\title{
The Bering Autonomous Target Detection
}

Jørgensen, John Leif; Denver, Troelz; Betto, Maurizio; Jørgensen, Peter Siegbjørn; Kurnaz, S.; Ince, F.; Onbassioglu, $\mathrm{S}$.

\section{Published in:}

Proceedings of the RAST Conference

Link to article, DOI:

10.1109/RAST.2003.1303918

Publication date:

2003

Document Version

Publisher's PDF, also known as Version of record

Link back to DTU Orbit

Citation (APA):

Jørgensen, J. L., Denver, T., Betto, M., Jørgensen, P. S., Kurnaz, S. (Ed.), Ince, F. (Ed.), \& Onbassioglu, S. (Ed.) (2003). The Bering Autonomous Target Detection. In Proceedings of the RAST Conference IEEE. https://doi.org/10.1109/RAST.2003.1303918

\section{General rights}

Copyright and moral rights for the publications made accessible in the public portal are retained by the authors and/or other copyright owners and it is a condition of accessing publications that users recognise and abide by the legal requirements associated with these rights.

- Users may download and print one copy of any publication from the public portal for the purpose of private study or research.

- You may not further distribute the material or use it for any profit-making activity or commercial gain

- You may freely distribute the URL identifying the publication in the public portal 


\title{
The Bering autonomous target detection
}

\author{
John L. Jørgensen, Troelz Denver, Maurizio Betto; Peter S. Jørgensen \\ Elektrovej, DTU - Building 327, 2800 Lyngby, Denmark, \\ Technical University of Denmark \\ Ørsted*DTU \\ Measurement and Instrumentation Systems
}

\begin{abstract}
An autonomous astroid target detection and tracking method has been develped. The method features near onmidirectionality and focus on high speed operations and completenes of search of the the near space rather than the traditional faint object search methods, employed presently at the larger telescopes. The method has proven robust in operation and is well suited for use onboard spacecrafts.

As develoment target for the method and the associated instrumentation the astroid research mission Bering has been used. Onboard a spacecraft, the autonomous detection is centered around the fully autonomous star tracker the Advanced Stellar Compass (ASC). One feature of this instrument is that potential targets are registered directly in true of date right ascension, declination and intensity, which greatly facilitates both tracking search and registering.

Results from ground and inflight tests are encouraging, both with respect to robustness, speed and accuracy, and demonstrates the span and range of applications of this techonlogy.
\end{abstract}

\section{INTRODUCTION}

The study of Asteroids and specifically Near Earth Objects (NEOs) is facing two severe problems which both are related to the way their initial detection and reobservation is made. The first is, that most of the Asteroid and NEO objects are faint due to their relatively small size compared to their distances, which bring the majority of these outside the envelope of standard astronomical telescope detection methods. The second is, that when an object eventually gets close enough, and in a pose that offers high luminosity, it is moving relatively fast which results in high proper motion rates. These problems are the main reasons why only a very small subset of Asteroids and NEOs have been observed and studied so far.

The Bering mission is designed exactly to circumvent this problem complex and will enable a much wider class of objects to be studied. The cruise phase of the planned Venus swing-by will offer excellent viewing conditions of NEOs with orbits inside the Earth, and the mission phase in the Asteroid beit will bring the "observatory" so close to the targets, that more that just the largest of objects can be studied.
Consequently, the Bering instrumentation is, unlike the astronomical telescopes, designed with focus on omni-directionality and high-speed operations, rather than faint objects observations.

A predicament Bering has in common with groundbased methods is the initial acquisition problem: The target is faint and far, which makes it appear similar to a multitude of other luminous objects such as stars, nebulae, galaxies etc. The only signifier that robustly discerns an Asteroid or NEO from the other deep space objects is the proper motion.

Onboard Bering, the initial target acquisition is performed using a special feature of the Advanced Stellar Compass (ASC) that constantly is scanning the firmament. The ASC is a star tracker that originally was developed to generate high accuracy attitude information based on analysis of star images. The ASC features fully autonomous operations and is presently flying on several spacecrafts from all over the world.

The ASC performs the attitude determination simply by acquiring a star image from a small $20 \mathrm{~mm}$ focal length low light camera. The image is then sifted for "stars", i.e. "centroided", and the centroid list is then compared to an onboard star catalogue. This process is extremely robust and accurate. A by-product of this process is a list of centroids, i.e. coordinates of luminous objects, which did not match an entry in the star catalogue. This, raw, list of non-stellar objects will contain un-mapped stars, galaxies, nebulae, and Asteroids.

Onboard Bering, this list of prospective targets is entered into a database. Since the ASCs are continuously scanning the firmament, several thousand observations of each object are achieved per hour. When entering a prospective target into the database, a special filter detect if an object at this specific position already exists, and if so, if the apparent proper motion is compatible with a solar system body. This procedure is greatly facilitated by the fact that the database entries are directly given in right ascension and declination. The result of this update filtering process is a list of objects with proper motions compatible with Asteroids and other close by objects.

The list of objects with high proper motion is then passed on to the onboard telescope scheduler/guider for more detailed observations as specified by the science requirements.

In the following the Bering initial target acquisition and registering methodology and the proper motion 
detection is described. Achievable detection limits and accuracies are given and the robustness discussed.

\section{THE $\mu$ ASC INSTRUMENT}

The autonomous target detection is performed by the miniature star tracker dubbed the $\mu \mathrm{ASC}$. The $\mu \mathrm{ASC}$ is physically divided into to two parts, a Data Processing Unit (DPU) and Camera. Head Unit (CHU). As indicated on the photo in figure 1, a DPU may drive up to four CHUs. This split unit philosophy permits the spacecraft designer to physically position the individual CHUs at locations optimised for the mission profile. The DPU shown in the picture is internally fully cold redundant which in combination with the multiple CHUs provide for unusually high reliability figures for the instrument complex.

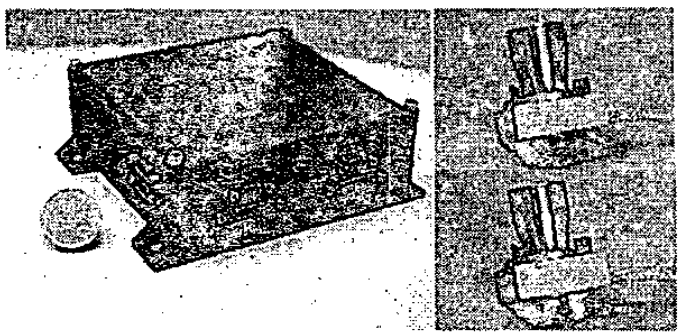

Fig. 1, The miniature DPU shown with two CHUs.

The intrinsic accuracy of an attitude measurement from a single CHU is better than one arcsecond at an integration time of $0.5 \mathrm{sec}$. This attitude is autonomously calculated based on all brighter stars in the Field of View (FOV) from that CHU. The basic operation of the instrument is described in details in [1].

The measured attitude may be construed as the rotation of the FOV from the firmament, mathematically represented as the quaternion $\mathrm{q} 1$. The right ascension and declination of any object in the image may be calculated from the rotation of the object from the centre of the FOV, represented by $q 2$, by using the resulting rotation $\mathrm{q} 2 \bullet \mathrm{q} l$.

\section{Basic OBJect Detection}

The powerful optics of the CHU reliably detects and calculates object positions down to visual magnitude 11 , $\mathrm{m}_{\mathrm{v}} 11$, at an integration time of one second. The standard integration time for simple attitude recovery onboard spacecrafts is typically chosen as a compromise between the need for timeliness and accuracy. Most star trackers employed in the attitude control loop of the spacecraft will therefore have an update rate from 1 to $4 \mathrm{~Hz}$. To cover these needs as well as supporting imaging of fainter objects, the integration time may be changed from 32 seconds to $1 / 16 \mathrm{sec}$ at any time.

The attitude accuracy is approximately proportional to the square root of the integration time, whereas the lower object detection limit is linearly proportional with integration time. This means, that the accuracy at, say, 16 seconds integration time is 0.25 arcseconds and, that the lower object detection limit is $m_{\mathrm{v}}$ 14. These numbers have been confirmed by on ground tests.

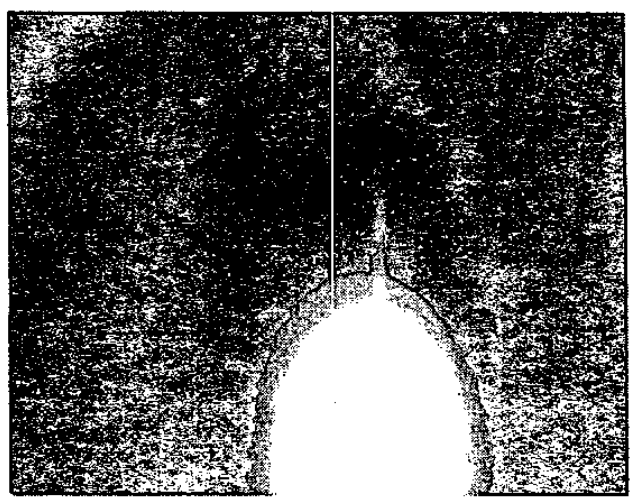

Fig. 2, The blooming effect from long integration times on the effective image area, here the Moon at approximately $m_{v}-5$.

Although larger integration times than $16 \mathrm{sec}$ are easily achievable, the processing becomes less straightforward at higher integration times, partly because of the chance of local blooming of the object by a nearby brighter image, partly because of the rapidly increasing demands posed to the attitude control of the spacecraft. So rather than pursuing increased integration time to meet science demands for deeper objects, we have chosen an increased focal length. The scale laws for focal length is roughly proportionality between accuracy and focal length. The lower object detection limit also scale inversely with the $F \#$, i.e. the opening aperture to focal length ratio. Test confirms these numbers for focal lengths between $20 \mathrm{~mm}$ and $250 \mathrm{~mm}$, with a measured attitude noise as low as $50 \mathrm{~m}$ as for the latter at 1 second integration time.

\section{OBJECT SCREENING CATALOGUE}

The standard star catalogue used to robustly derive the attitude of a CHU under most operational conditions encountered onboard spacecrafts, is made up from the brightest 14.500 stars, i.e. complete to approximately $m_{v}$ 7.3. This catalogue is optimised towards operations as 2$4 \mathrm{~Hz}$.

Consequently, using this catalogue to identify stars too be removed from the list of detected objects, will resuit in that all fainter stars has to be processed and eliminated later so as not to enter and overload the catalogue of objects of interest.

In order to eliminate stellar and deep space objects as soon as possible in the process, we have chosen to use a large star catalogue, the object screening catalogue, solely used to eliminate these objects. This catalogue is included in the $\mu \mathrm{ASC}$ SW. 
The size of this larger catalogue depends on the science requirements to how faint objects must be searched. So far, we have implemented catalogues complete to approximately $\mathrm{m}_{\mathrm{v}} 11$ and 13 containing 125.000 and 2.5 mio objects.

Processing time of an image down to mv 11 typically takes $350 \mathrm{~ms}$, and $\mathrm{mv} 13$ takes $1300 \mathrm{~ms}$, from no attitude knowledge until the screened list of objects are ready at the output.

We have tried to include larger catalogues, however, for the above mentioned catalogues, we have been using the TYCHO2 catalogue [2] as source, since this catalogue is both relatively complete, have good magnitude information and excellent accuracy on the position of the objects. The only publicly available catalogue that goes deeper is the UNSO [3] that contain the 526 mio brightest objects. The average accuracy of this catalogue is rather low, in the range of arcseconds, the result of which is a large error circle around each stellar object. Within this circle object detection and tracking are lost due to the false linking to the star of the catalogue. The result is a loss of $0.2 \%$ of the night sky covered by the error circles of the stars, which in itself is not problematic. To this comes, that the objects must be tracked for a certain time span, to allow for an accurate estimation of the orbital elements. The proper motion of an object to be tracked may cause it to cross in front of one of the error circles of a star, at which time the object may be lost by the tracking algorithm or, that the detection tracking must be performed anew after the object has left the error circle. All in all the result is an increase in the effective loss from the low accuracy of the catalogue between 5 and $10 \%$ depending on the tangential velocities of the objects that must be covered.

Another problem here is the size of the screening catalogue, that becomes rather bulky: Since a fast decoding is required, due to the frequent look-up processes, the penalty for compressing the catalogue is large in terms of extra search and decompression processor loads. In a no, or slightly compressed catalogue, either range keying or bifurcation may be used to efficiently locating the interesting section with a processing time only slightly depending on the catalogue size, since the search key is accurate right ascension and declination figures.

The above described problem complex drives for a limited size of the screening catalogue. If the science requirements demand fainter objects to be included in the object detection phase, the screening catalogue must be augmented in some fashion. In [4] the physical properties and orbital elements of various sizes of objects are discussed. It is demonstrated, that for a detection limit of $\mathrm{m}_{\mathrm{v}} 15$, the typical proper motion of the object, at the time of first detcction, is in the range of 1-20"/sec. Instead of a screening catalogue that is complete to the detection limit, a screening filter, which only propagates objects with tangential velocities above a certain minimal rate, is found to be more proficient.
Another important aspect is that while detection of fainter objects are important for a variety of science demands to an asteroid mission, such as timely detection and good count statistics, the scientifically most important ones are those that happens to pass rather close by the space craft.

Such close objects will exhibit rather high proper motion rates, and will therefore pose relatively high demands to the rate handling capability of the telescope tracking and guidance equipment. Brighter moving objects also create another problem. Since they will cover large angular distances in short times, they will inevitable get so close to other luminous objects that a erroneous merging by the centroider algorithm is inevitable, for the time of closest passage. Such merging is not as much a problem for the tracking but more for the correct determination of the orbital elements.

For this reason, and to make optimum use for science observations for the time of close flyby, the centroider algorithm must be capable of spanning a vast range of object intensities, and to keep track of the object during all phases of the flyby. This problem is further augmented by the inevitable blooming, chromatism and other second order effects highly luminous objects has on fast optics.

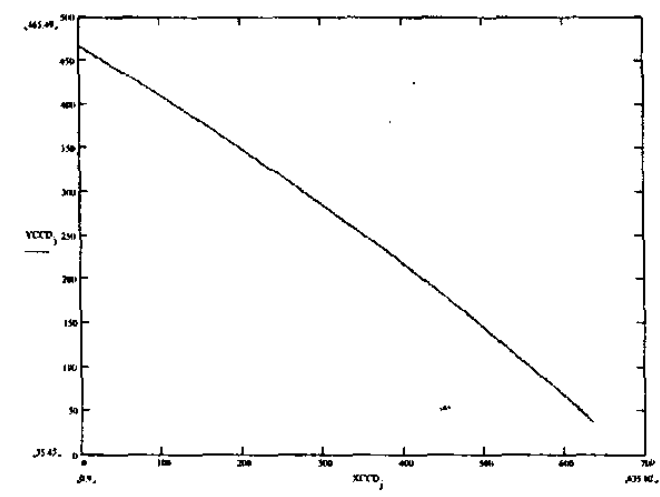

Fig. 3, Example of autonomous non-stellar object detection for a brighter target. The figure show the measured $C C D$ position of a relatively bright object. The target in the case shown was Mars. At a magnitude of -1 it was correctly identified and the position measured with an accuracy better than 4 " throughout the FOV.

Large but distant and therefore faint objects pose the opposite problem. These objects are easily centroided correctly, and easy to keep track of, but sepite their often high velocities, their proper motion is marginal due to their large distance. E.g. a $10 \mathrm{~km}$ object, moving with a tangential velocity of $25 \mathrm{~km} / \mathrm{sec}$ relative to the spacecraft, will only exhibit a proper motion of 0.25 arcsecond/sec at the detection limit if $\mathrm{m}_{\mathrm{v}} 7$ is assumed, and a mere 0.01 arcsecond/sec if a detection limit of $m_{y} 14$ is assumed, both for a reasonable phase angles and $1 \mathrm{AU}$ distance from the Sun. 


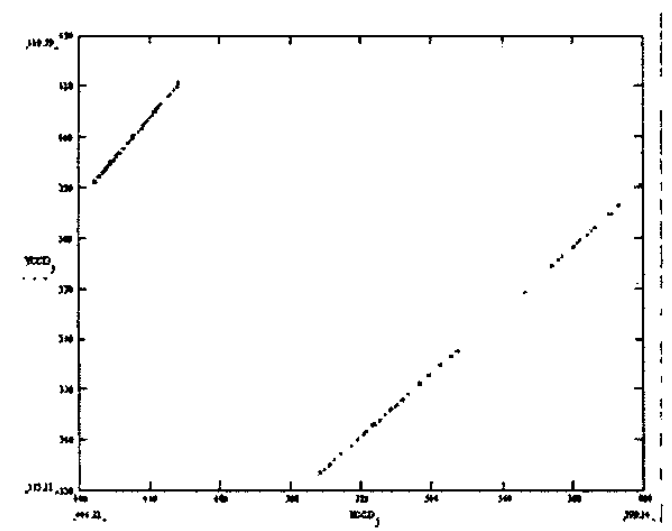

Fig. 4, Example of autonomous non-stellar object detection for a slow motion target. The figure show the measured CCD position of a relatively faint object. The target in the case shown was Uranus. At a magnitude of 6.7 it was correctly identified and the position measured with an accuracy better than 5 " throughout the FOV. The double line is caused by a repositioning of the $\mathrm{CCD}$ when Uranus moved outside the FOV.

\section{Prospective Target Catalogue}

At any given detection limit for the system, the number of far, large, but slowly moving objects must be assumed to be much higher than the smaller less distant ones.

As described above, these objects present the system with two problems. Firstly, the objects may not directly be discernible from stellar objects, either due to close object merging or due to incompleteness of the screening catalogue. Secondly these object exhibit small proper motions.

If such objects were entered unfiltered into the target database, this structure would soon be swamped with useless information, preventing efficient observation planning and autonomous target selection.

To overcome this problem, a catalogue of prospective targets (PTC) is used. This is designed to hold any detected, non recognized object for as long as it takes for it to accumulate sufficient proper motion for a reliable deletion or acceptance too the target database.

Here, again, the object position in right ascension and declination greatly facilitates the management of the catalogue. For each prospective object the position, intensity, time of first entry, last update, number of observations, measured motion speed and spread of the latter is registered.

Each time a prospective object is detected, the PTC is searched, and when a match is found, the object information is updated.

At regular intervals, the PTC must be scrubbed, i.e. the catalogue is searched for dead entries, that is objects that bave not been updated for a specified time, and more important, for objects that have accumulated sufficient proper motion to be safely identified as targets.

An alternative scrubbing procedure would be to eliminate objects that have not accumulated sufficient proper motion within some limits. This approach would result in a somewhat smaller PTC, but at the same time result in a much faster de-fragmentation of the PTC.

De-fragmentation is a problem to any sorted catalogue where entries are removed or added dynamically. For reasons of search and update speed it needs to maintain a rather linear structure. The problem occurs when an entry has to be made at a point in the catalogue that has no further space allocated. Typically, this problem is alleviated via. so-called side sectors, i.e. sub-catalogues for the superfluous entries, this techniques provide for fast updating, but will as the number of subcatalogues increase decrease the search efficiency.

From the above it is obvious that the PTC will need a thorough cleanup, or de-fragmentation at intervals.

For a moderate detection limit, the object screening catalogue may be made complete to fainter objects, which will result in very few non-inieresting objects are entered into the PTC. Since such relatively bright objects will possess sizeable proper motion rates, the scrubbing may be performed hourly, or even every few minutes, which will result in that promising targets are detected very fast. Also, the moderate size of the PTC will make it possible to only perform the time consuming de-fragmentation at long intervals.

If the detection limit goes well beyond the object screening catalogue, however, the scrubbing must be performed much more infrequent. This is partly because of the associated processing overhead, partly because the minimal proper motions will require long sampling times to prevent against false deletion of large but distant targets. The de-fragmentation, despite being a linear process, will still have to be performed regularly to avoid too large a swap space overhead.

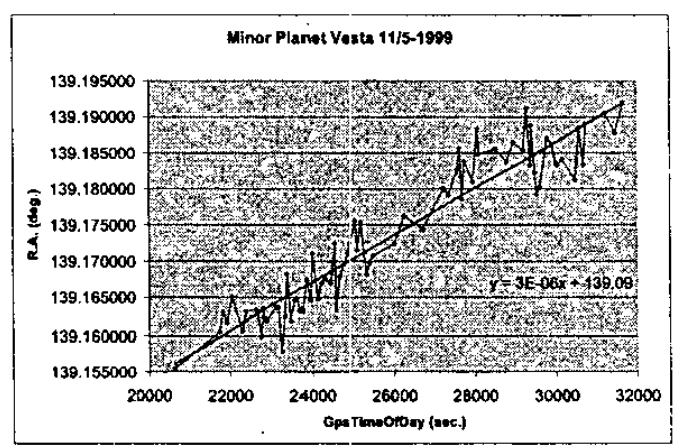

Fig 5, Example of the target isolation procedure applied on Vesta. The Object screening catalogue was limited to $\mathrm{m}_{\mathrm{v}} 9$, the target was approximately 7.7. Because of the limited size of the catalogue, scrubbing was performed once per $60 \mathrm{sec}$. The search area was $1 / 100$ of the nightsky. 


\section{TARGET CATALOGUE}

The final product of the autonomous detection procedure described above is a strongly filtered list of interesting objects, which it would be worth spending more time investigating in details. This list is labelled the "Target Catalogue", which contains the apparent proper motion, best guess of present position and magnitude for each entry.

This list obviously will also contain erroneously selected objects, that due to collision of circumstances have made it through the filtering, but these objects are few, at least for low to medium detection limits. For detection limits set for very faint objects, $\mathrm{m}_{\mathrm{v}} 14$ and fainter, the number of these erroneous objects may become excessive, and some quality index may have to be employed as well. I.e. based on the numbers stored in the PTC, entries that exhibit high scores, either intensities, proper motions of combinations of these, will get a higher score, than objects that just makes it past the detection criterion threshold.

Based on the spacecraft present attitude, and the projected attitude motion profile, the scan profile for the science telescope can be projected. From that knowledge, and the target catalogue, an observation programme may dynamically be generated and kept updated.

Since the target catalogue directly gives the required pointing direction towards to target to be observed, and since the telescope is directly and autonomously operate in right ascension and declination coordinates as described in [5], the search time for the science telescope is minimal. On average the time from target to target is expected to be 20 seconds, once on target the ASCFIT procedure [6] needed to cross calibrate the telescopes pointing direction to 50 mas accuracy may add up to 5 extra seconds. Assuming a necessary telescope time on target to be $100 \mathrm{sec}$ to determine the precise target characteristics the telescope will saturate (theoretical maximum) if more than 16.000 objects per day has to be observed.

The above performance estimate assumes an even distribution of targets and a low number of erroneous targets, which are very optimistic assumptions. Positive detection of a false target requires at least two visits to be detected and henceforth excluded. Furthermore, the target distribution must be expected to be concentrated in the galactic plane. Consequently, the effective targethandling rate will be somewhat less, and it will be further decreased by the time required to perform calibrations of the telescope spectral analyser, which may not always be performed when pointing out of the galactic plane. A more realistic estimate would be $16.000^{*} 0.5^{*} 0.3^{*} 0.95=$ 3800 targets per day.

\section{CONCLUSIONS}

A fully autonomous method to detect, isolate and create precise tracking information has been developed. The method has been made to robustly, and without time critical intervention from ground, operated onboard spacecrafts.

Throughout the development, focus has been on keeping processing power and memory demands low.

The method is highly scalable, from detecting small relatively bright objects fast, to relatively faint objects with associated observation schemes ranging over days.

All critical parts of the method has been successfully tested and verified via ground tests in realistic settings, using known solar system bodies.

\section{REFERENCES}

[1] J.L Jørgensen et al, "MicroASC A Miniature Star Tracker", in proceedings of Small Satellites for Earth. Observation IV $\left(4^{\text {th }} L A A\right), 2003$, p 157-162.

[2] E. Heg et al., "Construction and verification of the Tycho-2 Catalogue", Astronomy and Astrophysics, vol. 357, p.367-386, March 2000.

[3] D.G. Monet, "The USNO-B Catalog", Astronomical Journal, vol. 125 (2) , p. 984-93, 2003.

[4] R. Michelsen et al., "Asteroid and NEA Detection Models", in these proceedings, 2003.

[5] T. Denver et al., "The Bering Target Tracking Instrumentation", in these proceedings, 2003.

[6] P.S. Jørgensen et al., "ASCfit - Automatic Stellar Coordinate fitting package", in ASP conference Series, ASADD XI Conference, vol. 281, 2002. 